PROCEEDINGS OF THE

AMERICAN MATHEMATICAL SOCIETY

Volume 137, Number 12, December 2009, Pages 4219-4225

S 0002-9939(09)10044-8

Article electronically published on August 3, 2009

\title{
A WAVELET CHARACTERIZATION FOR THE DUAL OF WEIGHTED HARDY SPACES
}

\author{
MING-YI LEE, CHIN-CHENG LIN, AND YING-CHIEH LIN
}

(Communicated by Hart F. Smith)

\begin{abstract}
We define the weighted Carleson measure space $C M O_{w}^{p}$ using wavelets, where the weight function $w$ belongs to the Muckenhoupt class. Then we show that $C M O_{w}^{p}$ is the dual space of the weighted Hardy space $H_{w}^{p}$ by using sequence spaces. As an application, we give a wavelet characterization of $B M O_{w}$.
\end{abstract}

\section{INTRODUCTION}

Meyer 4 described the Hardy space $H^{1}$ and $B M O$ via wavelets. He offered several characterizations of $H^{1}$ in terms of its decompositions with respect to wavelet bases, and characterized $B M O$ in terms of a Carleson condition on wavelet coefficients. A natural extension is to consider their weighted counterparts. In 2001, Garcia-Cuerva and Martell 22 gave a wavelet characterization of weighted Hardy spaces $H_{w}^{p}(\mathbb{R}), 0<p \leq 1$. In this article, we give a wavelet characterization for the dual of $H_{w}^{p}(\mathbb{R}), 0<p \leq 1$. In order to do this, we define the weighted Carleson measure space $C M O_{w}^{p}$ and two sequence spaces $s_{w}^{p}$ and $c_{w}^{p}$. We first show that $c_{w}^{p}$ is the dual of $s_{w}^{p}$ and then obtain that $C M O_{w}^{p}$ is the dual of $H_{w}^{p}$. As a consequence, $C M O_{w}^{1}$ is the same as $B M O_{w}$, and hence we succeed by an approach different from the one in [5] for the wavelet characterization of $B M O_{w}$.

Let $\psi$ be an orthonormal wavelet; that is, $\psi \in L^{2}(\mathbb{R})$ such that the system

$$
\psi_{j, k}(x):=2^{j / 2} \psi\left(2^{j} x-k\right), \quad j, k \in \mathbb{Z},
$$

is an orthonormal basis for $L^{2}(\mathbb{R})$. We define the operator $\mathcal{W}_{\psi}$ by

$$
\mathcal{W}_{\psi} f=\left\{\sum_{j, k \in \mathbb{Z}}\left|\left\langle f, \psi_{j, k}\right\rangle\right|^{2}\left|I_{j, k}\right|^{-1} \chi_{I_{j, k}}\right\}^{1 / 2}, \quad f \in L^{2}(\mathbb{R}),
$$

Received by the editors November 26, 2008, and, in revised form, May 4, 2009.

2000 Mathematics Subject Classification. Primary 42B30, 42C40.

Key words and phrases. BMO , orthonormal wavelets, weighted Carleson measure spaces, weighted Hardy spaces.

The first author was supported by NSC of Taiwan under Grant \#NSC 97-2115-M-008-005.

The second and third authors were supported by NSC of Taiwan under Grant \#NSC 97-2115M-008-021-MY3.

(C)2009 American Mathematical Society Reverts to public domain 28 years from publication 
where $I_{j, k}=\left[2^{-j} k, 2^{-j}(k+1)\right]$. Denoting by $\mathcal{D}$ the set of all dyadic intervals $I_{j, k}$ with $j, k \in \mathbb{Z}$, and letting $\psi_{I_{j, k}}=\psi_{j, k}$, we can also write

$$
\mathcal{W}_{\psi} f=\left\{\sum_{I \in \mathcal{D}}\left|\left\langle f, \psi_{I}\right\rangle\right|^{2}|I|^{-1} \chi_{I}\right\}^{1 / 2} .
$$

Henceforth, we always use $I$ and $J$ to denote dyadic intervals. In what follows, we shall work exclusively with the one-dimensional case. For $\alpha \geq 1$, we say that $\psi$ belongs to the regularity class $\mathcal{R}^{\alpha}$ if $\psi \in C^{[\alpha]}$ and there exist positive constants $C, r, \varepsilon$ satisfying

(i) $\int_{\mathbb{R}} x^{n} \psi(x) d x=0$ for all $0 \leq n \leq[\alpha]-1$,

(ii) $|\psi(x)| \leq \frac{C}{(1+|x|)^{1+[\alpha]+r}} \quad$ for all $x \in \mathbb{R}$,

(iii) $\left|\psi^{(n)}(x)\right| \leq \frac{C}{(1+|x|)^{\alpha+\varepsilon}}$ for all $x \in \mathbb{R}$ and $0 \leq n \leq[\alpha]$.

Here $[\alpha]$ denotes the greatest integer not greater than $\alpha$.

The weight functions mentioned in this article refer to the Muckenhoupt $A_{q}$ weights. A weight $w \geq 0$ belongs to the class $A_{q}, 1<q<\infty$, if there is a constant $C>0$ such that

$$
\left(\int_{I} w(x) d x\right)\left(\int_{I} w(x)^{-1 /(q-1)} d x\right)^{q-1} \leq C|I|^{q} \quad \text { for any interval } I \subset \mathbb{R} .
$$

The class $A_{1}$ consists of weights $w$ satisfying for some $C>0$ that

$$
\frac{1}{|I|} \int_{I} w(x) d x \leq C \cdot \underset{x \in I}{\operatorname{essinf}} w(x) \quad \text { for any interval } I \subset \mathbb{R}
$$

and $A_{\infty}:=\bigcup_{1 \leq q<\infty} A_{q}$. For $w \in A_{\infty}$, denote by $q_{w}:=\inf \left\{q>1: w \in A_{q}\right\}$ the critical index of $w$. We use $w(E)$ to denote the weighted measure $\int_{E} w(x) d x$.

Let $\varphi \in \mathcal{S}$ satisfy $\int_{\mathbb{R}} \varphi(x) d x=1$. The maximal function $f^{*}$ is defined by

$$
f^{*}(x)=\sup _{r>0}\left|f * \varphi_{r}(x)\right|,
$$

where $\varphi_{r}(x)=r^{-1} \varphi(x / r), r>0$. The weighted Hardy spaces $H_{w}^{p}$ consist of those tempered distributions $f \in \mathcal{S}^{\prime}$ for which $f^{*} \in L_{w}^{p}$ with $\|f\|_{H_{w}^{p}}=\left\|f^{*}\right\|_{L_{w}^{p}}$. We refer readers to [1, 3] for the details about $A_{q}$ and $H_{w}^{p}$.

The following theorem was proved by Garcia-Cuerva and Martell [2].

Theorem A. Let $0<p \leq 1$ and $w \in A_{\infty}$. If $\psi \in \mathcal{R}^{\alpha}$ is an orthonormal wavelet with $\alpha \geq q_{w} / p$, then there exist two constants $0<c \leq C<\infty$ such that

$$
c\|f\|_{H_{w}^{p}} \leq\left\|\mathcal{W}_{\psi} f\right\|_{L_{w}^{p}} \leq C\|f\|_{H_{w}^{p}} .
$$

Definition. For $0<p \leq 1$ and $w \in A_{\infty}$, let $\psi \in \mathcal{R}^{\alpha}$ be an orthonormal wavelet with $\alpha \geq q_{w} / p$. The weighted Carleson measure space $C M O_{w}^{p}$ is the set of all $g \in L_{\text {loc }}^{1}$ satisfying

$$
\|g\|_{C M O_{w}^{p}}:=\sup _{J \in \mathcal{D}}\left\{\frac{1}{w(J)^{\frac{2}{p}-1}} \sum_{I \subset J}\left|\left\langle g, \psi_{I}\right\rangle\right|^{2} \frac{|I|}{w(I)}\right\}^{1 / 2}<\infty .
$$


Remark 1. If $w \equiv$ constant and $p=1$, then the above definition reduces to the Carleson condition that characterizes $B M O$ (cf. 4, p. 154]). Theorem A implies that the wavelet characterization of $H_{w}^{p}$ is independent of the choice of $\psi$, and hence, by the following Theorem 1, the definition of $C M O_{w}^{p}$ is independent of the choice of $\psi$, too.

We now state our main result as follows.

Theorem 1. For $0<p \leq 1$ and $w \in A_{\infty}$, let $\psi \in \mathcal{R}^{\alpha}$ be an orthonormal wavelet with $\alpha \geq q_{w} / p$. The dual of $H_{w}^{p}$ is $C M O_{w}^{p}$ in the following sense.

(a) For each $g \in C M O_{w}^{p}$, there is a linear functional $\ell_{g}$, initially defined on $H_{w}^{p} \cap L^{2}$, which has a continuous extension to $H_{w}^{p}$ and $\left\|\ell_{g}\right\| \leq C\|g\|_{C M O_{w}^{p}}$.

(b) Conversely, every continuous linear functional $\ell$ of $H_{w}^{p}$ can be realized as $\ell=\ell_{g}$ with some $g \in C M O_{w}^{p}$ and $\|g\|_{C M O_{w}^{p}} \leq C\|\ell\|$.

It is known that the dual space of $H_{w}^{1}$ is

$$
B M O_{w}=\left\{f \in L_{\text {loc }}^{1}: \sup _{\text {interval } Q} \frac{1}{w(Q)} \int_{Q}\left|f(x)-f_{Q}\right| d x<\infty\right\}
$$

for $w \in A_{\infty}$, and the dual space of $H_{w}^{p}, 0<p<1$, is

$$
\begin{aligned}
\left\{\frac{f(x)}{w(x)} \in L_{\mathrm{loc}}^{r^{\prime}}(w(x) d x):\right. & \left(\int_{Q}\left|\frac{f(x)-P_{Q}(x)}{w(x)}\right|^{r^{\prime}} \frac{w(x) d x}{w(Q)}\right)^{1 / r^{\prime}} \\
& \left.\leq C w(Q)^{1 / p-1} \text { for any bounded interval } Q\right\}
\end{aligned}
$$

for $w \in A_{r}, 1 \leq r<\infty$, where $f_{Q}=\frac{1}{|Q|} \int_{Q} f(x) d x$ and $P_{Q}$ is the unique polynomial of degree $\leq\left[q_{w} / p\right]-1$ such that $\int_{Q}\left(f(x)-P_{Q}(x)\right) x^{k} d x=0$ for $k=0,1, \cdots$, $\left[q_{w} / p\right]-1$ (see [1]). Thus, we have a wavelet characterization of $B M O_{w}$ and a continuous characterization of $C M O_{w}^{p}$ as follows.

Corollary 2. Let $0<p \leq 1$ and $w \in A_{r}, 1 \leq r \leq \infty$. Also let $\psi \in \mathcal{R}^{\alpha}$ be an orthonormal wavelet with $\alpha \geq q_{w} / p$.

(a) For $p=1$ and $w \in A_{\infty}, f \in B M O_{w}$ if and only if its wavelet coefficients $\left\langle f, \psi_{I}\right\rangle$ satisfy Carleson's condition:

$$
\sup _{J \in \mathcal{D}} \frac{1}{w(J)} \sum_{I \subset J}\left|\left\langle f, \psi_{I}\right\rangle\right|^{2} \frac{|I|}{w(I)} \leq C .
$$

(b) For $0<p<1$ and $w \in A_{r}, 1 \leq r<\infty, f$ satisfies (11) if and only if $f \in C M O_{w}^{p}$.

Remark 2. When the wavelet $\psi$ has compact support, the above characterization of $B M O_{w}$ was given by $\mathrm{Wu}[5]$. Here we offer a different but simpler approach.

\section{Sequence spaces}

In this section, we introduce two sequence spaces $s_{w}^{p}$ and $c_{w}^{p}, 0<p \leq 1$.

Definition. Let $0<p \leq 1$ and $w \geq 0$ be a weight function. The sequence space $s_{w}^{p}$ is defined to be the collection of all complex-valued sequences

$$
s_{w}^{p}=\left\{\left\{s_{I}\right\}:\left\|\left\{s_{I}\right\}\right\|_{s_{w}^{p}}:=\left\|\left(\sum_{I}\left|s_{I}\right|^{2}|I|^{-1} \chi_{I}\right)^{1 / 2}\right\|_{L_{w}^{p}}<\infty\right\} .
$$


Similarly, $c_{w}^{p}$ is defined to be the collection of all complex-valued sequences

$$
c_{w}^{p}=\left\{\left\{t_{I}\right\}:\left\|\left\{t_{I}\right\}\right\|_{c_{w}^{p}}:=\sup _{J \in \mathcal{D}}\left(\frac{1}{w(J)^{\frac{2}{p}-1}} \sum_{I \subset J}\left|t_{I}\right|^{2} \frac{|I|}{w(I)}\right)^{1 / 2}<\infty\right\} .
$$

Theorem 3. Let $0<p \leq 1$ and $w \in A_{\infty}$. The dual of $s_{w}^{p}$ is $c_{w}^{p}$ in the following sense.

(a) For each $\left\{t_{I}\right\} \in c_{w}^{p}$, the linear functional $\left\{s_{I}\right\} \mapsto \sum_{I} s_{I} \cdot \overline{t_{I}}$ is continuous on $s_{w}^{p}$.

(b) Conversely, every continuous linear functional on $s_{w}^{p}$ arises as in (a) with a unique element $\left\{t_{I}\right\}$ of $c_{w}^{p}$.

Moreover, the norm of $\left\{t_{I}\right\}$ as a linear functional on $s_{w}^{p}$ is equivalent to its $c_{w}^{p}$-norm.

Proof. (a) Given $\left\{t_{I}\right\} \in c_{w}^{p}$, it suffices to show that

$$
\left|\sum_{I} s_{I} \cdot \overline{t_{I}}\right| \leq C\left\|\left\{s_{I}\right\}\right\|_{s_{w}^{p}}\left\|\left\{t_{I}\right\}\right\|_{c_{w}^{p}} \quad \text { for all }\left\{s_{I}\right\} \in s_{w}^{p} .
$$

For $\left\{s_{I}\right\} \in s_{w}^{p}$, write

$$
\Omega_{k}=\left\{x \in \mathbb{R}: S(x):=\left(\sum_{I}\left|s_{I}\right|^{2}|I|^{-1} \chi_{I}(x)\right)^{1 / 2}>2^{k}\right\}
$$

and

$$
B_{k}=\left\{I: w\left(I \cap \Omega_{k}\right)>\frac{1}{2} w(I) \quad \text { and } \quad w\left(I \cap \Omega_{k+1}\right) \leq \frac{1}{2} w(I)\right\} .
$$

Then

$$
\left|\sum_{I} s_{I} \cdot \overline{t_{I}}\right|=\left|\sum_{k} \sum_{\tilde{I} \in B_{k}} \sum_{\substack{I \subset \tilde{I} \\ I \in B_{k}}} s_{I} \cdot \overline{t_{I}}\right| \leq \sum_{k} \sum_{\tilde{I} \in B_{k}} \sum_{\substack{I \subset \tilde{I} \\ I \in B_{k}}}\left|s_{I}\right|\left|\overline{\bar{I}_{I}}\right|,
$$

where $\tilde{I}$ 's are the maximal dyadic intervals in $B_{k}$. Applying the inequality $\|\cdot\|_{\ell^{1}} \leq$ $\|\cdot\|_{\ell^{p}}$, we get

$$
\begin{aligned}
\sum_{k} \sum_{\tilde{I} \in B_{k}} \sum_{\substack{I \subset \tilde{I} \\
I \in B_{k}}}\left|s_{I}\right|\left|\bar{t}_{I}\right| & \leq \sum_{k} \sum_{\tilde{I} \in B_{k}}\left(\sum_{\substack{I \subset \tilde{I} \\
I \in B_{k}}}\left|s_{I}\right|^{2} \frac{w(I)}{|I|}\right)^{1 / 2}\left(\sum_{\substack{I \subset \tilde{I} \\
I \in B_{k}}}\left|t_{I}\right|^{2} \frac{|I|}{w(I)}\right)^{1 / 2} \\
& \leq\left\{\sum_{k} \sum_{\tilde{I} \in B_{k}}\left(\sum_{\substack{I \subset \tilde{I} \\
I \in B_{k}}}\left|s_{I}\right|^{2} \frac{w(I)}{|I|}\right)^{p / 2}\left(\sum_{\substack{I \subset \tilde{I} \\
I \in B_{k}}}\left|t_{I}\right|^{2} \frac{|I|}{w(I)}\right)^{p / 2}\right\}^{1 / p} \\
& \leq\left\|\left\{t_{I}\right\}\right\|_{c_{w}^{p}}\left\{\sum_{k} \sum_{\tilde{I} \in B_{k}} w(\tilde{I})^{1-p / 2}\left(\sum_{\substack{I \subset \tilde{I} \\
I \in B_{k}}}\left|s_{I}\right|^{2} \frac{w(I)}{|I|}\right)^{p / 2}\right\}^{1 / p}
\end{aligned}
$$

Write

$$
\widetilde{\Omega}_{k}=\left\{x \in \mathbb{R}: M_{w}\left(\chi_{\Omega_{k}}\right)(x)>1 / 2\right\},
$$

where $M_{w}$ is the weighted Hardy-Littlewood maximal function defined by

$$
M_{w} f(x)=\sup _{\text {interval } Q \ni x} \frac{1}{w(Q)} \int_{Q}|f(x)| w(x) d x .
$$


Then $I \subset \widetilde{\Omega}_{k}$ for any $I \in B_{k}$. Since the $\tilde{I}$ 's are mutually disjoint dyadic intervals, $\sum_{\tilde{I} \in B_{k}} w(\tilde{I}) \leq w\left(\widetilde{\Omega}_{k}\right)$. We then apply Hölder's inequality to obtain

$$
\left|\sum_{I} s_{I} \cdot \overline{t_{I}}\right| \leq\left\|\left\{t_{I}\right\}\right\|_{c_{w}^{p}}\left\{\sum_{k} w\left(\widetilde{\Omega}_{k}\right)^{1-p / 2}\left(\sum_{I \in B_{k}}\left|s_{I}\right|^{2} \frac{w(I)}{|I|}\right)^{p / 2}\right\}^{1 / p} .
$$

We claim that

$$
\sum_{I \in B_{k}}\left|s_{I}\right|^{2} \frac{w(I)}{|I|} \leq C 2^{2 k} w\left(\widetilde{\Omega}_{k}\right)
$$

$M_{w}$ is of weak type $(1,1)$ with respect to $w(x) d x$, so $w\left(\widetilde{\Omega}_{k}\right) \leq C w\left(\Omega_{k}\right)$ and the claim gives

$$
\begin{aligned}
\left|\sum_{I} s_{I} \cdot \overline{t_{I}}\right| & \leq C\left\|\left\{t_{I}\right\}\right\|_{c_{w}^{p}}\left\{\sum_{k} 2^{k p} w\left(\widetilde{\Omega}_{k}\right)\right\}^{1 / p} \\
& \leq C\left\|\left\{t_{I}\right\}\right\|_{c_{w}^{p}}\left\{\sum_{k} 2^{k p} w\left(\Omega_{k}\right)\right\}^{1 / p} \\
& \leq C\left\|\left\{t_{I}\right\}\right\|_{c_{w}^{p}}\|S\|_{L_{w}^{p}} \\
& =C\left\|\left\{t_{I}\right\}\right\|_{c_{w}^{p}}\left\|\left\{s_{I}\right\}\right\|_{s_{w}^{p}} .
\end{aligned}
$$

To prove the claim, by the definitions of $S(x)$ and $B_{k}$, we have

$$
\int_{\widetilde{\Omega}_{k} \backslash \Omega_{k+1}} S^{2}(x) w(x) d x \leq 2^{2 k+2} w\left(\widetilde{\Omega}_{k}\right)
$$

and

$$
\begin{aligned}
\int_{\widetilde{\Omega}_{k} \backslash \Omega_{k+1}} S^{2}(x) w(x) d x & \geq \int_{\widetilde{\Omega}_{k} \backslash \Omega_{k+1}} \sum_{I \in B_{k}}\left|s_{I}\right|^{2}|I|^{-1} \chi_{I}(x) w(x) d x \\
& =\sum_{I \in B_{k}}\left|s_{I}\right|^{2} \frac{w\left(I \cap\left(\widetilde{\Omega}_{k} \backslash \Omega_{k+1}\right)\right)}{|I|} \\
& \geq \frac{1}{2} \sum_{I \in B_{k}}\left|s_{I}\right|^{2} \frac{w(I)}{|I|}
\end{aligned}
$$

(b) Clearly, every $\ell \in\left(s_{w}^{p}\right)^{\prime}$ is of the form

$$
\ell\left(\left\{s_{I}\right\}\right)=\sum_{I} s_{I} \overline{t_{I}}, \quad\left\{s_{I}\right\} \in s_{w}^{p},
$$

where $\left\{t_{I}\right\}$ is a certain sequence. Fix a dyadic interval $J$. Let $S_{J}=\{I \in \mathcal{D}: I \subset J\}$ and define a measure $\nu$ on $S_{J}$ by

$$
d \nu(I)=\frac{|I|}{w(J)^{\frac{2}{p}-1}} \quad \text { for } \quad I \in S_{J}
$$


By duality,

$$
\begin{gathered}
\left(\frac{1}{w(J)^{\frac{2}{p}-1}} \sum_{I \subset J}\left|t_{I}\right|^{2} \frac{|I|}{w(I)}\right)^{1 / 2}=\left\|\left\{t_{I} \frac{1}{w(I)^{\frac{1}{2}}}\right\}\right\|_{\ell^{2}\left(S_{J}, d \nu\right)} \\
\quad=\sup _{\left\|\left\{s_{I}\right\}\right\|_{\ell^{2}\left(S_{J}, d \nu\right)} \leq 1}\left|\sum_{I \subset J} s_{I} \overline{\bar{t}_{I}} \frac{|I|}{w(J)^{\frac{2}{p}-1} w(I)^{\frac{1}{2}}}\right| \\
\quad \leq\|\ell\| \sup _{\left\|\left\{s_{I}\right\}\right\|_{\ell^{2}\left(S_{J}, d \nu\right)} \leq 1}\left\|\left\{s_{I} \frac{|I|}{w(J)^{\frac{2}{p}-1} w(I)^{\frac{1}{2}}}\right\}\right\|_{s_{w}^{p}} .
\end{gathered}
$$

For $\left\{s_{I}\right\} \in \ell^{2}\left(S_{J}, d \nu\right)$, Hölder's inequality yields

$$
\begin{aligned}
\left\|\left\{s_{I} \frac{|I|}{w(J)^{\frac{2}{p}-1} w(I)^{\frac{1}{2}}}\right\}\right\|_{s_{w}^{p}} & =\frac{1}{w(J)^{\frac{2}{p}-1}}\left\{\int_{J}\left(\sum_{I \subset J}\left|s_{I}\right|^{2} \frac{|I|}{w(I)} \chi_{I}(x)\right)^{p / 2} w(x) d x\right\}^{1 / p} \\
& \leq\left\{\frac{1}{w(J)^{\frac{2}{p}-1}} \int_{J} \sum_{I \subset J}\left|s_{I}\right|^{2} \frac{|I|}{w(I)} \chi_{I}(x) w(x) d x\right\}^{1 / 2} \\
& =\left\|\left\{s_{I}\right\}\right\|_{\ell^{2}\left(S_{J}, d \nu\right)},
\end{aligned}
$$

and hence

$$
\sup _{\left\|\left\{s_{I}\right\}\right\|_{\ell^{2}\left(S_{J}, d \nu\right)} \leq 1}\left\|\left\{s_{I} \frac{|I|}{w(J)^{\frac{2}{p}-1} w(I)^{\frac{1}{2}}}\right\}\right\|_{s_{w}^{p}} \leq 1 .
$$

Taking the supremum over $J \in \mathcal{D}$ in (2), we obtain $\left\|\left\{t_{I}\right\}\right\|_{c_{w}^{p}} \leq\|\ell\|$.

\section{Proof of the MAIN THEOREM}

In this section we show that Theorem 1 follows as a consequence of Theorem 3 , Let $\psi \in \mathcal{R}^{\alpha}, \alpha \geq 1$, be an orthonormal wavelet. Define a map $P$ from the family of complex sequences into $\mathcal{S}^{\prime}$ by

$$
P\left(\left\{s_{I}\right\}\right)=\sum_{I} s_{I} \psi_{I}
$$

Define another map $L$ from function space into the family of complex sequences by

$$
L(f)=\left\{\left\langle f, \psi_{I}\right\rangle\right\}
$$

such that all $\left\langle f, \psi_{I}\right\rangle$ 's are well defined. Figure 1 illustrates the relationship among $s_{w}^{p}, c_{w}^{p}, H_{w}^{p}$, and $C M O_{w}^{p}$. Then $\left.P \circ L\right|_{L^{2}}$ is the identity on $L^{2}$. For $0<p \leq 1$ and

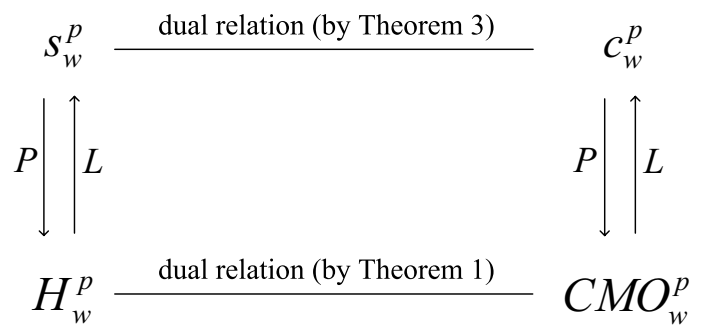

FiguRE 1. Diagram for spaces and maps

$w \in A_{\infty}$ with critical index $q_{w}$, if $\alpha \geq q_{w} / p$, then Theorem A yields

$$
\|\{L(f)\}\|_{s_{w}^{p}} \leq C\|f\|_{H_{w}^{p}} \quad \text { for } f \in H_{w}^{p} \cap L^{2}
$$


and

$$
\left\|P\left(\left\{s_{I}\right\}\right)\right\|_{H_{w}^{p}} \leq C\left\|\mathcal{W}_{\psi} P\left(\left\{s_{I}\right\}\right)\right\|_{L_{w}^{p}}=C\left\|\left\{s_{I}\right\}\right\|_{s_{w}^{p}} \quad \text { for }\left\{s_{I}\right\} \in s_{w}^{p} .
$$

By the definitions of $c_{w}^{p}$ and $C M O_{w}^{p}$,

$$
\|\{L(g)\}\|_{c_{w}^{p}}=\|g\|_{C M O_{w}^{p}} \quad \text { for } g \in C M O_{w}^{p}
$$

and

$$
\left\|P\left(\left\{t_{I}\right\}\right)\right\|_{C M O_{w}^{p}}=\left\|\left\{t_{I}\right\}\right\|_{c_{w}^{p}} \quad \text { for }\left\{t_{I}\right\} \in c_{w}^{p} .
$$

Proof of Theorem 1. For $g \in C M O_{w}^{p}$, define a linear functional $\tilde{\ell}_{g}$ by

$$
\tilde{\ell}_{g}(f)=\langle L(f), L(g)\rangle \quad \text { for } f \in H_{w}^{p} \cap L^{2} .
$$

By (3), (5), and Theorem 3 ,

$$
\left|\tilde{\ell}_{g}(f)\right| \leq C\|L(f)\|_{s_{w}^{p}}\|L(g)\|_{c_{w}^{p}} \leq C\|f\|_{H_{w}^{p}}\|g\|_{C M O_{w}^{p}} \quad \text { for } f \in H_{w}^{p} \cap L^{2} .
$$

Since $H_{w}^{p} \cap L^{2}$ is dense in $H_{w}^{p}$, the map $\tilde{\ell}_{g}$ can be extended to a continuous linear functional $\ell_{g}$ on $H_{w}^{p}$ satisfying $\left\|\ell_{g}\right\| \leq C\|g\|_{C M O_{w}^{p}}$.

Conversely, let $\ell \in\left(H_{w}^{p}\right)^{\prime}$ and set $\ell_{1}=\ell \circ P$ on $s_{w}^{p}$. It follows from (4) that $\ell_{1} \in\left(s_{w}^{p}\right)^{\prime}$. By Theorem 3, there exists $\left\{t_{I}\right\} \in c_{w}^{p}$ such that

$$
\ell_{1}\left(\left\{s_{I}\right\}\right)=\sum_{I} s_{I} \cdot \overline{t_{I}} \quad \text { for }\left\{s_{I}\right\} \in s_{w}^{p},
$$

and $\left\|\left\{t_{I}\right\}\right\|_{c_{w}^{p}} \approx\left\|\ell_{1}\right\| \leq C\|\ell\|$. For $f \in H_{w}^{p} \cap L^{2}$, we have

$$
\ell(f)=\ell_{1} \circ L(f)=\sum_{I}\left\langle f, \psi_{I}\right\rangle \overline{t_{I}}=\langle L(f), L(g)\rangle,
$$

where $g=\sum_{I} t_{I} \psi_{I}$. This shows that $\ell=\ell_{g}$, and (6) gives

$$
\|g\|_{C M O_{w}^{p}}=\left\|\left\{t_{I}\right\}\right\|_{c_{w}^{p}} \leq C\|\ell\| .
$$

Hence, the proof is finished.

\section{REFERENCES}

1. J. Garcia-Cuerva, Weighted $H^{p}$ spaces, Dissertationes Math. 162 (1979), 1-63. MR.549091 (82a:42018)

2. J. Garcia-Cuerva and J. M. Martell, Wavelet characterization of weighted spaces, J. Geom. Anal. 11 (2001), 241-264. MR.1856178 (2002h:42039)

3. J. García-Cuerva and J. L. Rubio de Francia, Weighted Norm Inequalities and Related Topics, North-Holland, Amsterdam, 1985. MR807149 (87d:42023)

4. Y. Meyer, Wavelets and Operators, Cambridge Studies in Advanced Mathematics, Vol. 37, Cambridge University Press, Cambridge, 1992. MR.1228209 (94f:42001)

5. S. Wu, A wavelet characterization for weighted Hardy spaces, Rev. Mat. Iberoamericana 8 (1992), 329-349. MR1202414 (94i:42027)

Department of Mathematics, National Central University, Chung-Li, Taiwan 320 , Republic OF China

E-mail address: mylee@math.ncu.edu.tw

Department of Mathematics, National Central University, Chung-Li, Taiwan 320 , Republic of China

E-mail address: clin@math.ncu.edu.tw

Department of Mathematics, National Central University, Chung-Li, Taiwan 320, Republic of China

E-mail address: linyj@math.ncu.edu.tw 